\title{
Correlation between cervical excisional procedures and the risk of preterm birth in subsequent pregnancies
}

\author{
Cezara Diana MUGESCU ${ }^{1}$, Brindusa Ana CIMPOCA-RAPTIS ${ }^{1}$, Anca Marina CIOBANU ${ }^{1,2}$, \\ Radu BOTEZATU ${ }^{1,2}$, Corina GICA ${ }^{1}$, Gheorghe PELTECU ${ }^{1,2}$, \\ Anca Maria PANAITESCU ${ }^{1,2}$, Nicolae GICA $^{1,2}$ \\ ${ }^{1}$ Filantropia Clinical Hospital, Bucharest, Romania \\ 2"Carol Davila" University of Medicine and Pharmacy, Bucharest, Romania
}

\section{ABSTRACT}

Cervical excisional procedures stand as an important risk factor for preterm birth (PTB) in subsequent pregnancies in various articles in the medical literature. We performed an evidence-based review of the obstetrical outcomes succeeding excisional procedures for cervical dysplasia as follows: we reviewed 16 studies published between 2015 and 2020 that assessed whether there is an association between excisional cervical procedures and unfavorable obstetrical outcomes. Nevertheless, there are several other aspects of the problem, taken into account as possibly responsible for increasing the risk of $P T B$, as the grade of the dysplasia, the effect of depth/length/volume of the excised cone specimen, prophylactic procedures for the prevention of PTB, age, interval conization-pregnancy. The majority of the authors suggested an association between excisional procedures and preterm birth or other obstetrical events. However, some of them did not find statistically significant data in order to strongly affirm a correlation between conization and PTB, or, on the contrary, disproved the given hypothesis. There is no consensus regarding cervical excisions as being an individual risk factor for PTB, therefore more studies need to be conducted in order to come to a strong, universal conclusion.
\end{abstract}

Keywords: cervix uteri, dysplasia, excisional procedure, premature delivery

\section{INTRODUCTION}

More than 550,000 women all over the world are diagnosed with cervical cancer every year, according to World Health Organization [1]. The same organization states that, globally, cervical cancer is the fourth most common cancer in women and Romania has the highest incidence and mortality rate of cervical cancer in Europe $[2,3]$. In our country, cervical cancer is the second most frequent cancer, after breast cancer [2]. Also, it is the most frequent diagnosed cancer in pregnant women [4].
The implementation of screening programs has led to a significant decrease in cervical cancer incidence [5], but on the other hand, due to the higher addressability of women to these programs, the incidence of premalignant lesions has risen [5], especially in younger, childbearing aged women [6].

Cervical intraepithelial neoplasia (CIN) is a premalignant lesion characterized by abnormal proliferation of the parabasal cells induced by infection with highrisk human papilloma virus (hr-HPV) that could potentially show progression to invasive carcinoma if left un- 
treated $[7,8]$. There are excisional procedures, such as large loop excision of the transformed zone (LLETZ), loop electrosurgical excision procedure (LEEP), needle excision of the transformation zone (TETZ) and cold knife conization (CKC) $[6,7]$ and ablative procedures, such as cryotherapy [7]. Histologically, these premalignant lesions could be classified as follows: low-grade squamous intraepithelial lesions (CIN1) and high-grade squamous intraepithelial lesions (CIN2 and CIN3) [9]. The aim of managing this condition is to both prevent possible progression to invasive cancer and avoid overtreatment of lesions that could naturally regress [10]. Therefore, American Society for Colposcopy and Cervical Pathology (ASCCP) recommends observation for $\mathrm{CIN} 1$ and excisional treatment for CIN 2 and 3 (over ablative procedures) [11].

There are several studies in medical literature that suggest, and even prove, the hypotheses that surgical procedures on the cervix may lead to adverse pregnancy outcomes, including preterm birth, miscarriage [5], low birth weight $[5,9,12,13]$. These outcomes occur as a consequence of shorter length of the cervix during pregnancy after an excisional procedure, which leads to mechanical weakness $[14,15]$, but also because of the immunomodulation related to HPV infection affecting biochemical pathways to parturition [14], compromised integrity of the cervical stroma and collagen alterations, or changes in the vaginal flora associated with HPV infection [12].

Every year, almost 15 million babies are born preterm globally, which means almost 1 in 10 births are preterm [16]. Causes of preterm births are multiple, among which multiple pregnancies, infectious or chronic conditions (high blood pressure and diabetes), genetic influences or previous surgery on cervix [16].

A relatively common procedure performed in order to prevent premature labor is prophylactic cerclage, but the efficacy of this method is debatable [17].

\section{RESULTS OF PREVIOUS STUDIES}

In 2015, Miller et al. [18] published an article based on a four years cohort study, which proved that women who underwent cervical excisional procedures have an increased risk for preterm birth. They divided all women who met the inclusion criteria into three categories (patients with no history of dysplasia, with history of dysplasia, but no excisional procedures and women who had prior cervical procedures) and assessed the risk of PTB in each of these categories. They also studied whether history of dysplasia alone is associated with the risk of PTB. Hence, the frequency of PTB among patients in the first two categories was $6.4 \%$ and $6.5 \%$ respectively, whereas amongst patients with prior cervical procedures was $8.4 \%$. Their main findings were that cervical dysplasia, in the absence of cervical procedures, does not increase the risk for PTB.

A Slovenian study conducted by Jancar et al. [5] and published in 2016 revealed a considerably higher risk for PTB in patients with history of excisional cervical procedures: $12.8 \%$ in women who had CKC, $8.2 \%$ in women who had LLETZ, whilst among patients with no history of excisional procedures the rate of PTB was only $4.1 \%$. Another interesting fact observed by the Slovenian team was the increasing number of pregnancies in women with history of conization. They put this on the account of postponement of reproduction and the introduction of National screening program for early cancer detection in Slovenia.

Another study that claims a higher risk for PTB in women with prior cervical excisional procedures was led by a Norwegian team [13]. Their article published in 2016, revealed an incidence of 9.7\% PTB among women with history of cervical excisional procedures, compared to only $5.3 \%$ in the group of patients with no previous treatment. Bjorge et al. also found an association of the treatment for cervical dysplasia before pregnancy and spontaneous abortion: $0.5 \%$ in comparison to $0.2 \%$.

The association between PTB and conisation is also supported by Heinonen et al. [9]. In their article $6.7 \%$ of women treated with LEEP and $5.2 \%$ of women with no history of LEEP delivered prematurely. They analyzed the risk of preterm birth before and after the diagnosis of CIN1 and concluded that it is associated with LEEP itself rather than with the diagnosis of CIN. Due to the spontaneous regression rate and the growing risk for PTB, LEEP should be avoided as treatment for CIN1, especially in young patients.

Although the majority of studies uphold the hypothesis (that surgical procedures of the cervix increase the risk of PTB, Liverani et al. [8] reported an incidence of only $2.4 \%$ preterm births in their study on 501 women who underwent a LEEP procedure. These data were unexpectedly lower than their anticipation and lower than those previous reported data in the developed countries.

Similarly, a retrospective study performed in Belgium and published in 2017 [19], outlined no significant difference concerning the risk of PTB between the group of women previously treated for cervical dysplasia and those not treated (5\% versus 3.3\%). The Leuven- 2 study could not prove a considerable association between preterm birth and conization as opposed to the first one.

Furthermore, Zebitay et al. [20] conducted a multicenter trial study on a cohort of Turkish women who gave birth over a period of 7 years. In this study there was only one case of PTB which represented a rate of $5 \%$, while in the control group the incidence of PTB was 
$1.29 \%$. There is a need to emphasize the additional risk factors for PTB that the woman in the study had: she had a previous premature delivery. Thereby, they concluded that the significant number of term deliveries after conisation is due to the absence of additional risk factors for PTB.

In 2018, Wittmaack et al. [21] led a retrospective cohort study to assess whether cervical excisional procedures increase the risk of PTB. Their results prove this hypothesis: out of 505 women who underwent cervical procedures in the past, $31.3 \%$ delivered prematurely, while in the group of patients with no history of conization or LEEP, $22.8 \%$ gave birth preterm. One of the possible explanations for preterm births after cervical excisional procedures refers to the inflammatory response caused by the microbial pathogens which ascend through the cervix, response that leads to a release of prostaglandins and eventual cervical ripening and uterine contractions [22].

Another study conducted by Frega et al. [23] and published in 2018 evaluated the incidence of PTB among women with history of cervical excisional procedures. Out of 468 patients who got pregnant after conization, $9.4 \%$ delivered prematurely, a prevalence considered as very low by the authors. They also assessed the impact of the dimensions of the cervical tissue excised, as well as the effect of vaginal infections and their persistency on the pregnancy. The authors demonstrated that the depth and the volume of the excised tissue is directly related with the risk of PTB. In addition, vaginal infections and their persistency during pregnancy after proper antibiotic therapy increases the risk of PTB, probably because of the alterations of the cervical mucus consequent to conizations, resulting in diminished antibacterial capacity and enabling the bacterial passage via cervix.

Papoutsis et al. [24] compared obstetric outcomes in women with history of LLETZ versus cold coagulation. Although the difference was not notable confronting the result between the previous mentioned categories ( $8.9 \%$ vs. $6.7 \%)$, it is significant when comparing the incidence of preterm births among the general population with the incidence amongst women with history of cervical procedures (3.85\% vs. $7.8 \%$ ). The authors assessed, as well, the incidence of miscarriages and concluded an incidence of $8.89 \%$ overall miscarriages in general population versus $28 \%$ in women with previous cervical surgeries.

In an article published in 2019, Ribaldone et al. [12] analyzed the association between excisional treatment for CIN and several obstetric outcomes (such as preterm birth, PROM or PPROM). The risk of PTB was significantly higher among women with prior treatment for CIN: $33.13 \%$ vs. $6.6 \%$, with an incidence of $88.68 \%$ late pre-term deliveries. Also, the risk of PROM was higher in women with history of cervical excisional procedures (40\% versus $23.22 \%$ ). In summary, their data confirmed the relationship between excisional treatment for CIN and unfavorable obstetric outcomes (e.g., preterm birth, PROM).

\section{CORRELATION BETWEEN DIMENSION OF THE EXCISED CONE AND PRETERM BIRTH}

Multiple studies evaluated the connection between the cone length or volume of the excised tissue and the risk of preterm birth. Therefore, in 2015, Liverani et al. [8] observed that all women in their study who delivered prematurely and underwent a conization had a cone specimen of $15 \mathrm{~mm}$ length or more (3.9\% had a cone length of $15-19 \mathrm{~mm}$ versus $15.6 \%$ - minimum 20 $\mathrm{mm}$ length). Moreover, all cases of preterm birth were reported in women with a cone volume of $2 \mathrm{~cm} 3$ or more. Hence, they concluded that it has been shown there is a direct relation between the depth and volume of the excised tissue and the risk of preterm birth because the deeper and larger the cervical resection, the greater the risk. Likewise, van Velthoven et al. [19] stated that the risk of preterm birth is significantly higher in case of a cervical excision deeper than $10 \mathrm{~mm}$ compared with nontreated women ( $22.8 \%$ vs. $8.6 \%)$. At the same time, Frega et al. [23] found a correlation between the risk of preterm birth and the cone specimen length. The authors observed a considerably high rate of preterm births in women with higher dimensions of the excised cervical tissue. Ribaldone et al. [12] found a rate of preterm birth of $33.13 \%$ among women treated with conization before birth and also observed that the risk of PTB rises with a cone depth of minimum $14 \mathrm{~mm}$. Besides, the rate increases by $0.3 \%$ with every $\mathrm{mm}$ of removed cervical tissue.

\section{AGE: ARE YOUNGER PATIENTS MORE SUSCEPTIBLE TO PRETERM BIRTH?}

Chevreau et al. [25] had a different and interesting approach on this thesis, which is dividing women who underwent cervical conization in two groups, by age (under the age of 25 and older than 25 years), and then evaluating the risk of undesirable obstetrical events in each group. It is intriguing that the differences, in terms of total number of obstetrical events or late miscarriage, were not significant, but there was a considerable disparity regarding these events under 26 WA, as follows: $19 \%$ rate of obstetrical events under 26 weeks of amenorrhea (WA) in women under 25 years old versus $4.1 \%$ in women older than 25 and $19 \%$ incidence of preterm labor in women under 25 years old versus $0 \%$ 
in the second group. The authors associated this finding with a lower adherence to medical investigations after cervical excisional procedures in case of younger women. The most important process to be incriminated in this issue seems to be cervical incompetence. In 2013, D'Agostini et al. [26] pointed out that female cervix is a growing organ and thus, surgically intervening on an immature cervix could lead to a definitive altering of the growing process, leading further to cervical incompetence. All things considered, Chevreau [25] stated that young women with CIN should better be treated conservatively, with the proviso that they submit to a meticulous program of medical investigations.

\section{TIME INTERVAL FROM CERVICAL CONIZATION: WHEN WILL BE SAFE A FUTURE PREGNANCY?}

Van Velthoven et al. [19] reported a higher and increasing risk for preterm birth in case of an early pregnancy, mainly if it is 2-3 months apart from the cervical excisional procedure. Their study revealed a predisposition to higher risk of PTB in the event of a pregnancy sooner than 12 months from the conization compared to more than 12 months. One possible explanation could be the vulnerability of the cervix caused by the structural changes during the regeneration process after the excisional procedure, vulnerability that might lead to a weak capability of the cervix to support a pregnancy. On the other hand, Papoutsis et al. [24] reported a higher risk of early miscarriages in the first 17 months from the conization. They found evidence that during the first 3 to 6 months from the cervical procedures, the cervix is under a complex process of histological, immunological and biochemical changes. A more recent study conducted by Zhang et al. [6] stated a safe interval LEEP-pregnancy of 6 months and CKC-pregnancy of 9 months. They observed no difference in the pre-operation cervical length compared to 9 months after CKC procedure, respectively to 6 and 9 months after LEEP.

\section{CERCLAGE: DOES IT REALLY PREVENT PTB IN WOMEN WHO UNDERWENT CONIZATION?}

Even more intriguing findings came out in several studies concerning methods to prevent preterm birth. In 2016, Kindinger et al. [14] studied the effects of cervical cerclage of different suture material on the risk of PTB. Their study revealed a rate of PTB of $10 \%$, meaning 70 out of 725 women, and $34 \%$ out of these 70 women delivered prematurely despite of cervical cerclage. As previously mentioned, the authors compared different suture materials and it appeared that among patients who received braided cerclage the rate of PTB was significantly higher than in the group of women receiving monofilament cerclage ( $40 \%$ vs. $15 \%)$. They related the deficiency of cerclage success to the idea that the cerclage thread is a "foreign" material and thus, it may cause alterations of the vaginal flora and the local immune system. This was the first study to emphasize the advantages of monofilament cerclage in women with history of conization and its beneficial role concerning PTB prevention. To sum up, Kindinger et al concluded that using a monofilament suture for cervical cerclage successfully diminishes the risk of preterm birth, as opposed to braided suture.

Later on, Cho et al. [17] concluded in their article published in 2018 that prophylactic cervical cerclage in women with history of conization is not mandatory. This Korean study did not find any advantage of the cerclage, on the contrary, the rate of PTB among women with cerclage was higher than in the group of women without cerclage $(10.56 \%$ vs. $4.27 \%)$. The connection between the presence of the cerclage and the inflammatory response of the cervix, is stipulated too in this study as the main cause of the high risk of preterm birth. The authors explained that based on their results, cerclage should be taken into account in women who have additional risk factors for preterm birth besides history of conization.

Not lastly, Zhang et al. [6] found the use prophylactic cerclage amongst women who underwent cervical excisional procedures as controversial. In this study, the authors proceeded to insert the prophylactic cerclage in the group of patients who underwent CKC, during the same surgical time in order to stimulate cervical growth. Their results needed though more cases to state a valid conclusion.

\section{CIN TREATMENT IN PREGNANT WOMEN: IS IT POSSIBLE?}

One of the most provoking studies was conducted by Siegler et al. [27] and referred to the possibility of treating CIN2/3 during the first trimester of pregnancy. They encouraged a change of recommendations targeting women diagnosed with CIN during pregnancy. In this study were observed 93 women with CIN2/3 divided in two groups: the observation group, consisting of 50 women treated conservatively, and the intervention group, comprising 43 women who were exposed to LEEP during the first 15 weeks of pregnancy. There were 37 women who continued with their pregnancy after the LEEP procedure; out of these 37 women, 2 have delivered preterm (at 34 and 36 weeks) and 1 experienced miscarriage at 10 weeks. The authors encourage treatment of CIN diagnosed during pregnancy 
relying on the fact that cervical intraepithelial neoplasia could progress to invasive cancer during pregnancy. On the other hand, they also highlighted the idea that untreated women will not return for further investigation and treatment after delivery. In conclusion, Siegler et al. find necessary to be taken into account LEEP during the first trimester of pregnancy, in women older than 25. In addition, the authors suggest that any delay in diagnosing early or minimally invasive cervical cancer during pregnancy could lead to an invasive cancer and so the need of a radical hysterectomy.

\section{CONCLUSIONS}

It remains clear that the hypothesis according to which prior cervical excisional procedures poses as a risk factor for preterm birth rests still an important food for thought. We observed there are several mechanisms incriminated as responsible for preterm birth among women with history of cervical operations by the majority of authors. Therefore, remodeling of the cervical structure, ascension of microbial pathogens through the cervical canal and prostaglandin release caused by the inflammatory response leading to uterine contractions, alteration of the mucus barrier resulting in diminished antibacterial capacity and enabling the bacterial passage via cervix, represent the core of the preterm birth occurrence.

Nonetheless, previous studies did not come to an exclusive conclusion, some pleading in favor of conisation as being a certain risk factor for PTB, others against this idea, most certainly because of the different population groups examined, their different way of life and culture, the differences concerning medical follow-up or indications/contraindications in different states, or the extent of the studies. As a consequence, more studies, preferably prospective studies must be conducted, on significant lots of patients.

Conflict of interest: none declared Financial support: none declared

\section{REFERENCES}

1. https://www.who.int/health-topics/cervicalcancer\#tab=tab_1.

2. https://insp.gov.ro/sites/cnepss/wp-content/ uploads/2018/05/Analiza-de-situatie-Cancercol-uterin-2018.pdf.

3. https://www.iob.ro/ro/proiecte/screeningcancer-col-program-national/informatii-utile.

4. NCCN Guidelines Version 1.2021 - cervical cancer.

5. Jančar N, Mihevc Ponikvar B, Tomšič S. Cold-knife conisation and large loop excision of transformation zone significantly increase the risk for spontaneous preterm birth: a population-based cohort study. Eur J Obstet Gynecol Reprod Biol. 2016 Aug;203:245-9.

6. Zhang X, Tong J, Ma X, Yu H, Guan X, Li J, Yang J. Evaluation of cervical length and optimal timing for pregnancy after cervical conization in patients with cervical intraepithelial neoplasia: A retrospective study. Medicine (Baltimore). 2020 Dec 4;99(49):e23411.

7. WHO guidelines for treatment of cervical intraepithelial neoplasia 2-3 and adenocarcinoma in situ: cryotherapy, large loop excision of the transformation zone, and cold knife conization.

8. Liverani CA, Di Giuseppe J, Clemente N, Delli Carpini G, Monti E, Fanetti F, Bolis G, Ciavattini A. Length but not transverse diameter of the excision specimen for high-grade cervical intraepithelial neoplasia (CIN 2-3) is a predictor of pregnancy outcome. Eur J Cancer Prev. 2016 Sep;25(5):416-22.

9. Heinonen A, Gissler M, Paavonen J, Tapper $A M$, Jakobsson M. Risk of preterm birth in women with cervical intraepithelial neoplasia grade one: a population-based cohort study. Acta Obstet Gynecol Scand. 2018 Feb;97(2):135-141.

10. Wright JD. Cervical intraepithelial neoplasia: Management. Available at: https://www. uptodate.com/contents/cervicalintraepithelial-neoplasia-management.

11. Perkins RB, Guido RS, Castle PE, Chelmow D, et al.; 2019 ASCCP Risk-Based Management Consensus Guidelines Committee. 2019 ASCCP Risk-Based Management Consensus Guidelines for Abnormal Cervical Cancer Screening Tests and Cancer Precursors. J Low Genit Tract Dis. 2020 Apr;24(2):102-131.

12. Maina G, Ribaldone R, Danese S, Lombardo V, Cavagnetto C, Plazzotta C, Surico D. Obstetric outcomes in patients who have undergone excisional treatment for high-grade cervical squamous intra-epithelial neoplasia. Eur J Obstet Gynecol Reprod Biol. 2019 May;236:210-213.

13. Bjørge T, Skare GB, Bjørge L, Tropé A, Lönnberg S. Adverse Pregnancy Outcomes After Treatment for Cervical Intraepithelial Neoplasia. Obstet Gynecol. 2016 Dec;128(6):1265-1273.

14. Kindinger LM, Kyrgiou M, MacIntyre DA, Cacciatore S, Yulia A, Cook J, Terzidou V, Teoh TG, Bennett PR. Preterm Birth Prevention Post-Conization: A Model of Cervical Length Screening with Targeted Cerclage. PLoS One. 2016 Nov 3;11(11):e0163793.

15. Kyrgiou M, Athanasiou A, Paraskevaidi M, Mitra A, Kalliala I, Martin-Hirsch P, Arbyn M,
Bennett P, Paraskevaidis E. Adverse obstetric outcomes after local treatment for cervical preinvasive and early invasive disease according to cone depth: systematic review and meta-analysis. BMJ. $2016 \mathrm{Jul}$ 28;354:i3633.

16. https://www.who.int/news-room/fact-sheets/ detail/preterm-birth.

17. Cho GJ, Ouh YT, Kim LY, Lee TS, Park GU, Ahn KH, Hong SC, Oh MJ, Kim HJ. Cerclage is associated with the increased risk of preterm birth in women who had cervical conization. BMC Pregnancy Childbirth. 2018 Jul 3;18(1):277.

18. Miller ES, Sakowicz A, Grobman WA. The association between cervical dysplasia, a short cervix, and preterm birth. Am J Obstet Gynecol. 2015 Oct;213(4):543.e1-4.

19. van Velthoven K, Poppe W, Verschuere H, Arbyn M. Pregnancy outcome after cervical conisation: A 2nd retrospective cohort study in the Leuven University Hospital. Eur J Obstet Gynecol Reprod Biol. 2017 Sep;216:224-231.

20. Zebitay AG, Güngör ES, Ilhan G, Çetin O, Dane C, Furtuna C, Atmaca FFV, Tuna M. Cervical Conization and the Risk of Preterm Birth: A Population-Based Multicentric Trial of Turkish Cohort. J Clin Diagn Res. 2017 Mar;11(3):QC21-QC24.

21. Wittmaack A, Dudley D, Boyle A. Maternal History of Cervical Surgery and Preterm Delivery: A Retrospective Cohort Study. J Womens Health (Larchmt). 2019 Nov;28(11):1538-1542.

22. Svare JA, Andersen LF, Langhoff-Roos J, Jensen ET, Bruun B, Lind I, Madsen $\mathrm{H}$. The 
relationship between prior cervical conization, cervical microbial colonization and preterm premature rupture of the membranes. Eur J Obstet Gynecol Reprod Biol. 1992 Oct 23;47(1):41-5.

23. Frega A, Santomauro M, Sesti F, Di Giuseppe J, Colombrino C, Marziani R, Catalano A, Pavone M, Leone C, Mallozzi M, D'Adamo E, Ciavattini A, Caserta D. Preterm birth after loop electrosurgical excision procedure (LEEP): how cone features and microbiota could influence the pregnancy outcome. Eur Rev Med Pharmacol Sci. 2018 Oct;22(20):7039-7044.
24. Papoutsis D, Underwood M, Parry-Smith W, Panikkar J. Early and late pregnancy outcomes in women treated with coldcoagulation versus LLETZ cervical treatment for cervical intraepithelial neoplasia; a retrospective cohort study. Arch Gynecol Obstet. 2018 Apr;297(4):1015-1025.

25. Chevreau J, Mercuzot A, Foulon A, Attencourt C, Sergent F, Lanta S, Gondry $\mathrm{J}$. Impact of Age at Conization on Obstetrical Outcome: A Case-Control Study. J Low Genit Tract Dis. 2017 Apr;21(2):97-101.
26. D'Agostini C, de Oliveira M, D'Souza-Li L. Comparison of cervical length in adult and adolescent nulliparae at mid-gestation. $J$ Pediatr Adolesc Gynecol. 2013 Aug;26(4):209-11.

27. Siegler E, Lavie O, Amit A, Vaknin Z, Auslander R, Blumenfeld Z; Israeli Colposcopy Network. Should the Risk of Invasive Cancer in Pregnancy and the Safety of Loop Electrosurgical Excision Procedure During the First 15 Weeks Change Our Practice? J Low Genit Tract Dis. 2017 Oct;21(4):299-303. 